\title{
Sufficient conditions for local equivalence of mappings
}

\author{
Piotr Migusio
}

\begin{abstract}
Let $f, g:\left(\mathbb{R}^{n}, 0\right) \rightarrow\left(\mathbb{R}^{m}, 0\right)$ be $C^{r+1}$ mappings and let $Z$ $=\left\{x \in \mathbb{R}^{n}: \nu(d f(x))=0\right\}, 0 \in Z, m \leq n$. We will show that if there exist a neighbourhood $U$ of $0 \in \mathbb{R}^{n}$ and constants $C, C^{\prime}>0$ and $k>1$ such that for $x \in U$

$$
\begin{aligned}
& \nu(d f(x)) \geq C \operatorname{dist}(x, Z)^{k-1}, \\
& \left|\partial^{s}\left(f_{i}-g_{i}\right)(x)\right| \leq C^{\prime} \nu(d f(x))^{r+k-|s|},
\end{aligned}
$$

for any $i \in\{1, \ldots, m\}$ and for any $s \in \mathbb{N}_{0}^{n}$ such that $|s| \leq r$, then there exists a $C^{r}$ diffeomorphism $\varphi:\left(\mathbb{R}^{n}, 0\right) \rightarrow\left(\mathbb{R}^{n}, 0\right)$ such that $f=g \circ \varphi$ in a neighbourhood of $0 \in \mathbb{R}^{n}$. By $\nu(d f)$ we denote the Rabier function.
\end{abstract}

Mathematics Subject Classification. Primary 58K40; Secondary 14B05.

Keywords. Diffeomorphism, $C^{r}$ equivalence, Right equivalence.

1. Introduction. Let $C^{k}(n)$ denote the space of $C^{k}$ function germs $\left(\mathbb{R}^{n}, 0\right) \rightarrow$ $\mathbb{R}$, where $k, n \in \mathbb{N}$ and let $C^{k}(n, m)=\left(C^{k}(n)\right)^{m}$ denote the space of $C^{k}$ mapping germs. In the sequel instead of a function germ (a mapping germ) we will write simply a function (a mapping). Recall that $C^{k}(n)$ is a local ring.

Let $f, g:\left(\mathbb{R}^{n}, 0\right) \rightarrow\left(\mathbb{R}^{m}, 0\right)$ be $C^{k}$ mappings. We say that $f$ and $g$ are $C^{r}$-right equivalent if there exists a $C^{r}$ diffeomorphism $\varphi:\left(\mathbb{R}^{n}, 0\right) \rightarrow\left(\mathbb{R}^{n}, 0\right)$ such that $f=g \circ \varphi$ in a neighbourhood of $0 \in \mathbb{R}^{n}$. Let $\mathbb{N}_{0}=\mathbb{N} \cup\{0\}$. A norm in $\mathbb{R}^{n}$ we denote by $|\cdot|$ and by $\operatorname{dist}(x, V)$ the distance of a point $x \in \mathbb{R}^{n}$ to a set $V \subset \mathbb{R}^{n}$.

In this paper we will prove a sufficient condition for $C^{r}$-right equivalence of mappings. The result which we obtain is a generalization of the following.

Theorem $1.1([8])$. Let $f, g:\left(\mathbb{R}^{n}, 0\right) \rightarrow(\mathbb{R}, 0)$ be $C^{r+1}$ functions, $r \in \mathbb{N}$. If $\nabla f(0)=0$ and if there exist a neigbourhood $U$ of $0 \in \mathbb{R}^{n}$ and a constant $C>0$ such that

This research was partially supported by NCN, Grant Number 2015/17/B/ST1/02637. 


$$
\left|\partial^{m}(g-f)(x)\right| \leq C|\nabla f(x)|^{r+2-|m|} \quad \text { for } x \in U
$$

and for any $m \in \mathbb{N}_{0}^{n}$ such that $|m| \leq r$, then $f$ and $g$ are $C^{r}$-right equivalent.

There exist two classical techniques to get a suitable diffeomorphism (both cases refer to a local equivalence).

The first originates from the theorem of Kuiper-Kuo $[4,5]$ and relies on constructing a vector field and integration of this field. Kuiper and Kuo used this technique to give a sufficient condition for a topological equivalence of functions with isolated singularities (higher classes of an equivalence of isolated singularities were considered by Takens [12]). Xu generalized this theorem for the case of non-isolated singularities [14]. Theorem 1.1 is on the one hand a counterpart of the theorem of $\mathrm{Xu}$ in the case of equivalence of higher classes $(r>0)$, on the other hand a counterpart of the theorem of Takens in the case of non-isolated singularities. It is worth pointing out that the theorem of Kuiper-Kuo and the theorem of $\mathrm{Xu}$ are formulated in the language of jets. Obviously, there arises a natural question what happens in the case of mappings with non-isolated singularities. Rodak, Spodzieja, and the author of this paper [9] and Bekka and Koike [1] independently took that topic and obtained sufficient conditions for topological equivalence without any assumption about singularities which are straightforward generalization of the theorem of $\mathrm{Xu}$.

Before we give the main theorem of this paper we have to introduce some notation. Let $X, Y$ be Banach spaces over $\mathbb{R}$. Let $L(X, Y)$ denote a Banach space of linear continuous mappings from $X$ to $Y$. For $A \in L(X, Y), A^{*}$ stands for the adjoint operator in $L\left(Y^{\prime}, X^{\prime}\right)$, where $X^{\prime}$ is the dual space of $X$. For $A \in L(X, Y)$ we put

$$
\nu(A)=\inf \left\{\left\|A^{*} \varphi\right\|: \varphi \in Y^{\prime},\|\varphi\|=1\right\},
$$

where $\|A\|$ is the norm of a linear mapping $A$ (see [10]). In the case of functions we have $\nu(d f)=|\nabla f|$, where $d f$ is the differential of $f$. The main result of this paper is the following

Theorem 1.2. Let $f, g:\left(\mathbb{R}^{n}, 0\right) \rightarrow\left(\mathbb{R}^{m}, 0\right)$ be $C^{r+1}$ mappings and let $Z=\{x \in$ $\left.\mathbb{R}^{n}: \nu(d f(x))=0\right\}, 0 \in Z, m \leq n$. If there exist a neighbourhood $U$ of $0 \in \mathbb{R}^{n}$ and constants $C, C^{\prime}>0$ and $k>1$ such that for $x \in U$

$$
\begin{aligned}
& \nu(d f(x)) \geq C \operatorname{dist}(x, Z)^{k-1}, \\
& \left|\partial^{s}\left(f_{i}-g_{i}\right)(x)\right| \leq C^{\prime} \nu(d f(x))^{r+k-|s|},
\end{aligned}
$$

for any $i \in\{1, \ldots, m\}$ and for any $s \in \mathbb{N}_{0}^{n}$ such that $|s| \leq r$, then $f$ and $g$ are $C^{r}$-right equivalent. In particular the mappings $f$ and $g$ are $C^{r}$ isotopic at zero.

For the definition of $C^{r}$ isotopy see Subsection 2.2. The above theorem is proved in the spirit of the theorem of Kuiper-Kuo. In comparison to Theorem 1.1 we had to add an extra condition (namely (1.1)) which is forced by tools used for the proof.

The second technique to get a diffeomorphism is Tougeron's implicit function theorem [13]. To formulate consequences of this theorem we need some definitions. 
Let $f \in C^{k+1}(n, m)$. By $\mathcal{M}_{f} C^{k}(n)$ we will denote an ideal in $C^{k}(n)$ generated by all maximal minors of the Jacobi matrix of $f$. By $\mathfrak{m} C^{k}(n)$ we mean maximal ideal in $C^{k}(n)$.

As a corollary of Tougeron's implicit function theorem we have

Theorem $1.3([2])$. Let $f, g:\left(\mathbb{R}^{n}, 0\right) \rightarrow\left(\mathbb{R}^{m}, 0\right)$ be $C^{r+2}$ mappings and let

$$
\mathcal{M}_{f} C^{r+1}(n) \subset \mathfrak{m} C^{r+1}(n) \text {. }
$$

If

$$
(g-f) C^{r+1}(n) \subset \mathfrak{m} C^{r+1}(n)\left(\mathcal{M}_{f} C^{r+1}(n)\right)^{2},
$$

then $f$ and $g$ are $C^{r}$-right equivalent.

The first technique is geometrical and the second one is algebraic. Results proved in [7, Main Theorem], [8, Theorem 3] show that we can substitute the geometrical condition by the algebraic. We partially do that in Corollary 4.1.

Moreover, in view of Lemma 2.7, results which we obtain in this paper are true in the complex case.

Additionally in the last section we show the following result.

Proposition 1.4. Let $f, g:\left(\mathbb{R}^{n}, 0\right) \rightarrow\left(\mathbb{R}^{m}, 0\right)$ be $C^{1}$ mappings with locally Lipschitz differentials $d f, d g:\left(\mathbb{R}^{n}, 0\right) \rightarrow L\left(\mathbb{R}^{n}, \mathbb{R}^{m}\right)$, let $Z=\left\{x \in \mathbb{R}^{n}: \nu(d f(x))\right.$ $=0\}$, and let $0 \in Z, m \leq n$. If there exist a neigbourhood $U \in \mathbb{R}^{n}$ and constants $C>0, C_{1} \in\left(0, \frac{1}{2}\right)$, and $k>1$ such that

$$
\begin{aligned}
& \nu(d f(x)) \geq C \operatorname{dist}(x, Z)^{k-1}, \\
& |f(x)-g(x)| \leq C \nu(d f(x))^{k}, \\
& \|d f(x)-d g(x)\| \leq C_{1} \nu(d f(x))^{k-1}
\end{aligned}
$$

for $x \in U$, then $f$ and $g$ are bi-lipschitz equivalent.

In the above theorem by bi-lipschitz equivalence we mean that there exists a Lipschitz mapping $\varphi:\left(\mathbb{R}^{n}, 0\right) \rightarrow\left(\mathbb{R}^{n}, 0\right)$, the inverse of which is also a Lipschitz mapping, such that $f=g \circ \varphi$ in some neighbourhood of $0 \in \mathbb{R}^{n}$.

\section{Preliminaries.}

2.1. The Rabier function. Let $X, Y$ be Banach spaces over $\mathbb{K}$, where $\mathbb{K}=\mathbb{R}$ or $\mathbb{K}=\mathbb{C}$. Let $L(X, Y)$ denote a Banach space of linear continuous mappings from $X$ to $Y$. For $A \in L(X, Y), A^{*}$ stands for the adjoint operator in $L\left(Y^{\prime}, X^{\prime}\right)$, where $X^{\prime}$ is the dual space of $X$. We begin by recalling some properties of the Rabier function (cf. [11]).

Lemma $2.1([6])$. Let $\Sigma$ be the set of operators $A \in L(X, Y)$ such that $A(X) \subset$ $Y, A(X) \neq Y$. Then

$$
\nu(A)=\operatorname{dist}(A, \Sigma), \quad A \in L(X, Y) .
$$

Lemma 2.2 ([10]). Let $A, B \in L(X, Y)$. Then $|\nu(A)-\nu(B)| \leq\|A-B\|$. In particular $\nu: L(X, Y) \rightarrow \mathbb{R}$ is Lipschitz.

From Lemma 2.2 we have 
Lemma 2.3. If $A, B \in L(X, Y)$, then

$$
\nu(A+B) \geq \nu(A)-\|B\| .
$$

Definition 2.4 ([3]). Let $\mathbf{a}=\left[a_{i j}\right]$ be the matrix of $A \in L\left(\mathbb{K}^{n}, \mathbb{K}^{m}\right), n$ $\geq m$. By $M_{I}(A)$, where $I=\left(i_{1}, \ldots, i_{m}\right)$ is any subsequence of $(1, \ldots, n)$, we denote an $m \times m$ minor of a given by columns indexed by $I$. Moreover, if $J=\left(j_{1}, \ldots, j_{m-1}\right)$ is any subsequence of $(1, \ldots, n)$ and $j \in\{1, \ldots, m\}$, then by $M_{J}(j)(A)$ we denote an $(m-1) \times(m-1)$ minor of a given by columns indexed by $J$ and with deleted $j$ th row (if $m=1$, we put $M_{J}(j)(A)=1$ ). Let

$$
\begin{aligned}
h_{I}(A) & =\max \left\{\left|M_{J}(j)(A)\right|: J \subset I, j=1, \ldots, m\right\}, \\
g^{\prime}(A) & =\max _{I} \frac{\left|M_{I}(A)\right|}{h_{I}(A)} .
\end{aligned}
$$

Here we put $0 / 0=0$. If $m=n$, we put $h_{I}=h$.

Lemma 2.5 ([3]). There exist $C_{1}, C_{2}>0$, such that for any $A \in L\left(\mathbb{K}^{n}, \mathbb{K}^{m}\right)$ we have

$$
C_{1} g^{\prime}(A) \leq \nu(A) \leq C_{2} g^{\prime}(A) .
$$

Corollary 2.6 ([11]). The function $g^{\prime}$ is continuous.

Lemma $2.7([\mathbf{6}])$. Assume that $X, Y$ are complex Banach spaces. Let $\Sigma_{\mathbb{C}}$ (resp. $\Sigma_{\mathbb{R}}$ ) be the set of non-surjective $\mathbb{C}$-linear (resp. $\mathbb{R}$-linear) continuous maps from $X$ to $Y$. Then for any continuous $\mathbb{C}$-linear map $A: X \rightarrow Y$,

$$
\operatorname{dist}\left(A, \Sigma_{\mathbb{C}}\right)=\operatorname{dist}\left(A, \Sigma_{\mathbb{R}}\right) .
$$

2.2. Isotopy. Let $\Omega \subset \mathbb{R}^{n}$ be a neighbourhood of $0 \in \mathbb{R}^{n}, Z \subset \mathbb{R}^{n}$ be a set such that $0 \in Z$ and let $r \in \mathbb{N}_{0}$.

We will say that a continuous mapping $H: \Omega \times[0,1] \rightarrow \mathbb{R}^{n}$ is a $C^{r}$ isotopy near $Z$ at zero if

(a) $H_{0}(x)=x$ for $x \in \Omega$ and $H_{t}(x)=x$ for $t \in[0,1]$ and $x \in \Omega \cap Z$,

(b) for any $t$ the mapping $H_{t}$ is a $C^{r}$ diffeomorphism,

where the mapping $H_{t}: \Omega \rightarrow \mathbb{R}^{n}$ is defined by $H_{t}(x)=H(x, t)$ for $x \in \Omega$, $t \in[0,1]$.

Let $f: \Omega_{1} \rightarrow \mathbb{R}^{m}, g: \Omega_{2} \rightarrow \mathbb{R}^{m}$ where $\Omega_{1}, \Omega_{2} \subset \mathbb{R}^{n}$ are neighbourhoods of $0 \in \mathbb{R}^{n}$ and let $Z \subset \mathbb{R}^{n}$ be a set such that $0 \in Z$. We call $f$ and $g C^{r}$ isotopic near $Z$ at zero if there exists a $C^{r}$ isotopy near $Z$ at zero $H: \Omega \times[0,1] \rightarrow \mathbb{R}^{n}$, $\Omega \subset \Omega_{1} \cap \Omega_{2}$, such that $f\left(H_{1}(x)\right)=g(x), x \in \Omega$.

2.3. Lemmas. The following two lemmas are from [8, Lemma 2] and [7, Corollary 1], respectively.

Lemma 2.8. Let $f:\left(\mathbb{R}^{n}, 0\right) \rightarrow\left(\mathbb{R}^{m}, 0\right)$ be a locally Lipschitz mappings. Then there exist a neighbourhood $U$ of $0 \in \mathbb{R}^{n}$ and a constant $C>0$ such that for any $x \in U,|f(x)| \leq C \operatorname{dist}\left(x, V_{f}\right)\left(V_{f}\right.$ denote zero set of $\left.f\right)$.

Let $\mathcal{Q}=\left(q_{1}, \ldots, q_{m}\right)$, where $q_{1}, \ldots, q_{m} \in C^{k}(n)$ and let $\mathcal{Q} C^{k}(n)$ denote an ideal in $C^{k}(n)$ generated by $q_{1}, \ldots, q_{m}$. 
Lemma 2.9. Let $f, g:\left(\mathbb{R}^{n}, 0\right) \rightarrow(\mathbb{R}, 0)$ be $C^{r+1}$ functions, $r \in \mathbb{N}$. If $(g-f) \in$ $\left(\mathcal{Q} C^{r}(n)\right)^{r+k}$, then there exist a neighbourhood $U$ of $0 \in \mathbb{R}^{n}$ and a constant $C>0$ such that

$$
\left|\partial^{m}(g-f)(x)\right| \leq C|\mathcal{Q}(x)|^{r+k-|s|} \quad \text { for } x \in U
$$

and for any $s \in \mathbb{N}_{0}^{n}$ such that $|s| \leq r$.

Lemma 2.10. Let $U$ be a neighbourhood of $0 \in \mathbb{R}^{n}, f: U \rightarrow \mathbb{R}$ be a $C^{r}$ function, and $Z=\left\{x \in \mathbb{R}^{n}: f(x)=0\right\}, r \in \mathbb{N}$. There exists a $C^{r-|\alpha|}$ function $w_{\alpha}$ such that

$$
\partial^{\alpha}\left(\frac{1}{f(x)}\right)=\frac{w_{\alpha}(x)}{f(x)} .
$$

for $x \in U \backslash Z$ and for any $\alpha \in \mathbb{N}_{0}^{n},|\alpha| \leq r$. Moreover, if there exists a constant $C>0$ such that $|f(x)| \geq C \operatorname{dist}(x, Z)^{k-1}$ for $x \in U \backslash Z$, then

$$
\left|\partial^{\alpha}\left(\frac{1}{f(x)}\right)\right| \leq \frac{C^{\prime}}{\operatorname{dist}(x, Z)^{k-1}} \quad \text { for } x \in U \backslash Z
$$

and for a constat $C^{\prime}>0$

Proof. To prove the first part we will carry out induction with respect to $|\alpha|$. For $|\alpha|=0$ the assertion is obvious. Assume that (2.1) holds for $|\alpha|=k$. Let $\gamma=\left(\alpha_{1}, \ldots, \alpha_{i}+1, \ldots, \alpha_{n}\right),|\alpha|+1=|\gamma|$. We have

$$
\begin{aligned}
\partial^{\gamma}\left(\frac{1}{f(x)}\right) & =\frac{\partial}{\partial x_{i}} \partial^{\alpha}\left(\frac{1}{f(x)}\right)=\frac{\partial}{\partial x_{i}}\left(\frac{w_{\alpha}(x)}{f(x)}\right)=\frac{\frac{\partial w_{\alpha}}{\partial x_{i}}(x) f(x)-w_{\alpha}(x) \frac{\partial f}{\partial x_{i}}(x)}{(f(x))^{2}} \\
& =\frac{1}{f(x)}\left(\frac{\partial w_{\alpha}}{\partial x_{i}}(x)-\frac{w_{\alpha}(x) \frac{\partial f}{\partial x_{i}}(x)}{f(x)}\right)=\frac{w_{\gamma}(x)}{f(x)}
\end{aligned}
$$

where $w_{\gamma}$ is a $C^{r-|\alpha|-1}$ function on $U \backslash Z$. To prove (2.2) it is sufficient to observe that $w_{\alpha}$ is at least continuous on $U \backslash Z$, therefore there exists a constant $C^{\prime}>0$ such that $\left|w_{\alpha}\right| \leq C^{\prime}$ for $x \in U \backslash Z$.

3. Proof of Theorem 1.2. Let $P=g-f=\left(P_{1}, \ldots, P_{m}\right)$ and let $U=\left\{x \in \mathbb{R}^{n}\right.$ : $|x|<\varepsilon\}$ be a neighbourhood such that $f$ and $g$ are defined. By Lemma 2.8 there exists a positive constant $A$ such that

$$
\nu(d f(x)) \leq A \operatorname{dist}(x, Z) \quad \text { for } x \in U .
$$

Consider the mapping $F: \mathbb{R} \times \mathbb{R}^{n} \rightarrow \mathbb{R}^{m}$,

$$
F(\xi, x)=f(x)+\xi P(x)
$$

Let us fix $\xi \in(-2,2)$. By (1.1), (1.2), Lemma 2.3, and Lemma 2.8 there exist $C_{1}, C_{1}^{\prime}>0$ such that for $x \in U$ we obtain

$$
\begin{aligned}
\nu\left(d_{x} F(\xi, x)\right) & =\nu(d f(x)+\xi d P(x)) \geq \nu(d f(x))-|\xi||d P(x)| \\
& \geq \nu(d f(x))-|\xi| C_{1} \nu(d f(x))^{r+k-1} \\
& \geq C_{1}^{\prime} \operatorname{dist}(x, Z)^{k-1} .
\end{aligned}
$$

Thus by Lemma 2.5 there exists $C^{\prime \prime}>0$ such that

$$
g^{\prime}\left(d_{x} F(\xi, x)\right) \geq C^{\prime \prime} \operatorname{dist}(x, Z)^{k-1}, \quad \xi \in(-2,2), \quad x \in U .
$$


Set $G=\{(\xi, x) \in \mathbb{R} \times U:|\xi|<2\}$. In the notation of Definition 2.4 we put

$$
A_{I}=\left\{(\xi, x) \in G: \frac{\left|M_{I}\left(d_{x} F(\xi, x)\right)\right|}{h_{I}\left(d_{x} F(\xi, x)\right)} \leq \frac{C^{\prime \prime}}{2} \operatorname{dist}(x, Z)^{k-1}\right\} .
$$

By Lemma 2.6 the sets $A_{I}$ are closed in $G$ and $(\mathbb{R} \times Z) \cap G \subset A_{I}$. From (3.3) we see that $\left\{G \backslash A_{I}: I\right\}$ is an open covering of $\left\{G \backslash A_{I}: I \in \mathfrak{I}\right\}$. Let $\left\{\delta_{I}: \mathbb{R}^{n} \rightarrow[0,1]: I \in \mathfrak{I}\right\}$ be a $C^{\infty}$ partition of unity associated to this covering.

Let us consider the following system of linear equations

$$
\left(d_{x} F(\xi, x)\right) W(\xi, x)^{T}=-P(x)^{T}
$$

with indeterminates $W(\xi, x)=\left(W_{1}(\xi, x), \ldots, W_{n}(\xi, x)\right)$ and parameters $(\xi, x) \in G$. Take any subsequence $I=\left(i_{1}, \ldots, i_{m}\right)$ of the sequence $(1, \ldots, n)$. For simplicity of notation we assume that $I=(1, \ldots, m)$. For all $(\xi, x) \in G$ such that $M_{I}\left(d_{x} F(\xi, x)\right) \neq 0$ we put $W^{I}(\xi, x)=\left(W_{1}^{I}(\xi, x), \ldots, W_{n}^{I}(\xi, x)\right)$, by

$$
\begin{aligned}
& W_{l}^{I}(\xi, x)=\sum_{j=1}^{m}\left(-P_{j}(x)\right)(-1)^{l+j} \frac{M_{I \backslash l}(j)\left(d_{x} F(\xi, x)\right)}{M_{I}\left(d_{x} F(\xi, x)\right)}, \quad l=1, \ldots, m, \\
& W_{l}^{I}(\xi, x)=0, \quad l=m+1, \ldots, n,
\end{aligned}
$$

where $I \backslash l=(1, \ldots, l-1, l+1, \ldots, m)$ for $l=1, \ldots, m$. Cramer's rule implies

$$
\left(d_{x} F(\xi, x)\right) W^{I}(\xi, x)^{T}=-P(x)^{T} .
$$

Let $W: G \rightarrow \mathbb{R}^{n}$ be the following mapping

$$
W(\xi, x)=\left\{\begin{array}{ll}
\sum_{\left\{I \in \mathfrak{I}: \delta_{I}(\xi, x) \neq 0\right\}} \delta_{I}(\xi, x) W^{I}(\xi, x) & \text { for }(\xi, x) \in G \backslash(\mathbb{R} \times Z) \\
0 & \text { for }(\xi, x) \in G \cap(\mathbb{R} \times Z)
\end{array} .\right.
$$

Obviously $W=\left(W_{1}, \ldots, W_{n}\right)$, where $W_{l}=\sum \delta_{I} W_{l}^{I}$.

Note that $\delta_{I} W^{I}$ is a $C^{r}$ mapping on $G \backslash(\mathbb{R} \times Z)$ (after suitable extension). Hence $W=\sum_{I} \delta_{I} W^{I}$ is also a $C^{r}$ mapping on $G \backslash(\mathbb{R} \times Z)$. It is easy to see, that $W$ satisfies the equation (3.4).

We will show that $W$ is a $C^{r}$ mapping. The proof of this fact we will carry out in three steps.

Step 1. The mapping $W$ is continuous in $G$. 
Indeed, from (1.1), the definitions of $A_{I}$, the choice of $P$, the above construction, and (3.1) there exists a constant $A^{\prime}>0$ such that

$$
\begin{aligned}
|W(\xi, x)| & \leq \sum_{\left\{I \in \mathfrak{I}: \delta_{I}(\xi, x) \neq 0\right\}} \delta_{I}(\xi, x)\left|W^{I}(\xi, x)\right| \\
& \leq \sum_{\left\{I \in \mathfrak{I}: \delta_{I}(\xi, x) \neq 0\right\}} \delta_{I}(\xi, x) \sqrt{n} \max _{l=1}^{m}\left|W_{l}^{I}(\xi, x)\right| \\
& \leq \sum_{\left\{I \in \mathfrak{I}: \delta_{I}(\xi, x) \neq 0\right\}} \delta_{I}(\xi, x) \sqrt{n} \sum_{j \in I}\left|P_{j}(x)\right| \frac{h_{I}\left(d_{x} F(\xi, x)\right)}{\left|M_{I}\left(d_{x} F(\xi, x)\right)\right|} \\
& \leq \sum_{\left\{I \in \mathfrak{I}: \delta_{I}(\xi, x) \neq 0\right\}} \delta_{I}(\xi, x) \sqrt{n} \sum_{j \in I} C^{\prime} \operatorname{dist}(x, Z)^{r+k} \frac{2}{C^{\prime \prime}} \frac{1}{\operatorname{dist}(x, Z)^{k-1}} \\
& =A^{\prime} \operatorname{dist}(x, Z)^{r+1} .
\end{aligned}
$$

The above inequality also holds for $(\xi, x) \in G \cap(\mathbb{R} \times Z)$, therefore $W$ is continuous in $G$.

Step 2. Let $\alpha=\left(\alpha_{0}, \ldots, \alpha_{n}\right) \in \mathbb{N}_{0}^{n+1}$ be such that $|\alpha| \leq r$, then there exists a constant $A^{\prime \prime}>0$ such that

$$
\left|\partial^{\alpha} W_{l}\right| \leq A^{\prime \prime} \operatorname{dist}(x, Z)^{r+1-|\alpha|} \quad \text { for }(\xi, x) \in G \backslash(\mathbb{R} \times Z) .
$$

Indeed, let us take $(\xi, x) \in G \backslash A_{I}$, from the Leibniz's rule we have

$$
\left|\partial^{\alpha} W_{l}^{I}(\xi, x)\right| \leq \sum_{j=1}^{m} \sum_{\beta \leq \alpha}\left(\begin{array}{l}
\alpha \\
\beta
\end{array}\right)\left|\partial^{\alpha-\beta}\left(P_{j}(x)\right)\right|\left|\partial^{\beta}\left(\frac{M_{I \backslash l}(j)\left(d_{x} F(\xi, x)\right)}{M_{I}\left(d_{x} F(\xi, x)\right)}\right)\right| .
$$

Diminishing $G$ if necessary, from Lemma 2.10 we obtain

$$
\left|\partial^{\beta}\left(\frac{M_{I \backslash l}(j)\left(d_{x} F(\xi, x)\right)}{M_{I}\left(d_{x} F(\xi, x)\right)}\right)\right| \leq \frac{A_{\beta}^{\prime \prime}}{\nu(d f(x))^{k-1}},
$$

for constants $A_{\beta}^{\prime}>0$. Therefore, from (3.5) we have

$$
\left|\partial^{\alpha} W_{l}^{I}(\xi, x)\right| \leq \sum_{j=1}^{m} \sum_{\beta \leq \alpha}\left(\begin{array}{l}
\alpha \\
\beta
\end{array}\right)\left|\partial^{\alpha-\beta}\left(P_{j}(x)\right)\right| \frac{A_{\beta}^{\prime \prime}}{\nu(d f(x))^{k-1}} .
$$

From (1.2) we have

$$
\left|\partial^{\alpha-\beta}\left(P_{j}(\xi, x)\right)\right| \leq C^{\prime} \nu(d f(x))^{r+k-|\alpha|+|\beta|}
$$

Finally, from (3.6), (3.7), and (3.1) we obtain

$$
\begin{aligned}
\left|\partial^{\alpha} W_{l}^{I}(\xi, x)\right| & \leq \sum_{j=1}^{m} \sum_{\beta \leq \alpha}\left(\begin{array}{l}
\alpha \\
\beta
\end{array}\right) C^{\prime}(\nu d f(x))^{r+k-|\alpha|+|\beta|} \frac{A_{\beta}^{\prime \prime}}{\operatorname{dist}(x, Z)^{k-1}} \\
& \leq \sum_{j=1}^{m} \sum_{\beta \leq \alpha}\left(\begin{array}{l}
\alpha \\
\beta
\end{array}\right) A A_{\beta}^{\prime \prime} C^{\prime} \operatorname{dist}(x, Z)^{r+k-|\alpha|+|\beta|-k+1} \\
& \leq A^{\prime \prime \prime} \nu(d f(x))^{r+1-|\alpha|}
\end{aligned}
$$


for some constant $A^{\prime \prime \prime}>0$ and for $(\xi, x) \in G \backslash A_{I}$.

Moreover, from above there exists a constant $A^{\prime \prime}>0$ such that for $(\xi, x) \in$ $G \backslash(\mathbb{R} \times Z)$

$$
\begin{aligned}
\left|W_{l}\right| & =\left|\partial^{\alpha} \sum_{\left\{I \in \mathfrak{I}: \delta_{I}(\xi, x) \neq 0\right\}} \delta_{I} W_{l}^{I}\right| \\
& \leq \sum_{\left\{I \in \mathcal{I}: \delta_{I}(\xi, x) \neq 0\right\}} \sum_{\beta \leq \alpha}\left(\begin{array}{l}
\alpha \\
\beta
\end{array}\right)\left|\partial^{|\alpha|-|\beta|} \delta_{I}\right|\left|\partial^{|\beta|} W_{l}^{I}\right| \\
& \leq A^{\prime \prime} \operatorname{dist}(x, Z)^{r+1-|\alpha|} .
\end{aligned}
$$

Step 3. Partial derivatives $\partial^{\alpha} W_{l}$ vanish for $(\xi, x) \in G \cap(\mathbb{R} \times Z)$ and $|\alpha| \leq r$.

Indeed, we will carry out induction with respect to $|\alpha|$. Let $t \in \mathbb{R}, x \in Z$, and let $x_{m}^{t}=\left(x_{1}, \ldots, x_{m}+t, \ldots, x_{n}\right)$. For $|\alpha|=0$ the hypothesis is obvious. Assume that the hypothesis is true for $|\alpha| \leq r-1$. Then from Step 2 we have

$$
\begin{aligned}
\frac{\left|\partial^{\alpha} W_{l}\left(\xi, x_{m}^{t}\right)-\partial^{\alpha} W_{l}(\xi, x)\right|}{|t|} & =\frac{\left|\partial^{\alpha} W_{l}\left(\xi, x_{m}^{t}\right)\right|}{|t|} \leq \frac{A^{\prime \prime} \operatorname{dist}\left(x_{m}^{t}, Z\right)^{r+1-|\alpha|}}{|t|} \\
& \leq \frac{A^{\prime \prime}|t|^{r+1-|\alpha|}}{|t|}=A^{\prime \prime}|t|^{r-|\alpha|}
\end{aligned}
$$

Since $r-|\alpha| \geq r-r+1=1$, we obtain $\partial^{\gamma} W_{l}(\xi, x)=0$ for $x \in Z$ and $|\gamma|=|\alpha|+1$. This completes Step 3 .

In summary from Step 1, 2, and 3 we obtain that $W_{l}$ are $C^{r}$ functions in $G$. Therefore, $W$ is a $C^{r}$ mapping in $G$.

Let us consider the following system of differential equations

$$
y^{\prime}=W(t, y) .
$$

Since $r \geq 1, W$ is at least of class $C^{1}$ on $G$, so it has a uniqueness of solutions property in $G$. Choose $(\xi, x) \in G$ and define $\varphi_{(\xi, x)}$ to be the maximal solution of $(3.8)$ such that $\varphi_{(\xi, x)}(\xi)=x$. Set $\Omega_{0}=\left\{x \in \mathbb{R}^{n}:\|x\|<r_{0}\right\}$, $\Omega_{1}=\left\{x \in \mathbb{R}^{n}:\|x\|<r_{1}\right\}$, where $r_{0}, r_{1}>0$. Since $0 \in Z$, the mapping $\varphi(\xi)=0, \xi \in \mathbb{R}$, is a solution of (3.8). Hence for sufficiently small $r_{0}, r_{1}$, for any $x \in \Omega_{0}$, the solution $\varphi_{(0, x)}$ is defined on $[0,1]$ and $\varphi_{(0, x)}(t) \in \Omega_{1}$ if $t \in[0,1]$ and for any $x \in \Omega_{1}$, the solution $\varphi_{(1, x)}$ is also defined on $[0,1]$. Let $H, \widetilde{H}: \Omega_{0} \times[0,1] \rightarrow \Omega_{1}$ be given by

$$
H(x, t)=\varphi_{(0, x)}(t), \quad \widetilde{H}(y, t)=\varphi_{(t, y)}(0) .
$$

The mappings $H, \widetilde{H}$ are well defined. Moreover one can extend these mappings to $C^{r}$ mappings on some open neighbourhood of $\Omega_{0} \times[0,1]$. Put $\Omega=\Omega_{1}$, $\Omega^{t}=\left\{y \in \mathbb{R}^{n}: \widetilde{H}(y, t) \in \Omega\right\}, t \in[0,1]$. By uniqueness of solutions of (3.8), for any $t$ we have $\widetilde{H}(H(x, t), t)=x, H(x, 0)=x, x \in \Omega$, and $H(\widetilde{H}(y, t))=y$, $y \in \Omega^{t}$. Moreover there exists a neighbourhood $\Omega^{\prime} \subset \mathbb{R}^{n}$ of 0 such that $\Omega^{\prime} \subset \Omega^{t}$ for any $t$. 
Finally, by (3.4) we have

$$
\frac{d}{d t} F\left(t, \varphi_{(\xi, x)}(t)\right)^{T}=P(x)^{T}+\left(d_{x} F\right)\left(t, \varphi_{(\xi, x)}(t)\right) W\left(t, \varphi_{(\xi, x)}(t)\right)^{T}=0,
$$

so, $F\left(t, \varphi_{(0, x)}(t)\right)=f(x)$ and consequently $f(H(x, 1))+t P(H(x, 1))=f(x)$ for $t \in[0,1]$ and $x \in \Omega^{\prime}$. This ends the proof.

4. Additional results. As an immediate consequence of Theorem 1.2 and Lemma 2.9 we have

Corollary 4.1. Let $f, g:\left(\mathbb{R}^{n}, 0\right) \rightarrow\left(\mathbb{R}^{m}, 0\right)$ be $C^{r+1}$ mappings and let $Z=\{x \in$ $\left.\mathbb{R}^{n}: \nu(d f(x))=0\right\}, 0 \in Z, m \leq n$. If there exist a neighbourhood $U$ of $0 \in \mathbb{R}^{n}$ and constants $C, C^{\prime}>0$ and $k>1$ such that for $x \in U$

$$
\begin{aligned}
& \nu(d f(x)) \geq C \operatorname{dist}(x, Z)^{k-1}, \\
& \left(g_{i}-f_{i}\right) \in\left(\nu(d f) C^{r}(n)\right)^{r+k}
\end{aligned}
$$

for any $i \in\{1, \ldots, m\}$, then $f$ and $g$ are $C^{r}$-right equivalent. In particular mappings $f$ and $g$ are $C^{r}$ isotopic at zero. By $(\nu(d f)) C^{r}(n)$ we mean the ideal in $C^{r}(n)$ generated by the Rabier function $\nu(d f)$.

Let us pay attention that despite Theorem 1.3 and Corollary 4.1 look similar, this result possesses an essential difference. Namely Theorem 1.3 is about $C^{r}$-right equivalence of $C^{r+2}$ mappings while Corollary 4.1 is about $C^{r}$-right equivalence of $C^{r+1}$ mappings. In fact in Theorem 1.2 and Corollary 4.1 we show slightly more. Namely that two mappings are $C^{r}$ isotopic.

Under previous notations, in the paper [9, Corollary 1.4] it was proved that under the following conditions

$$
\begin{aligned}
& \nu(d f(x)) \geq C \operatorname{dist}(x, Z), \\
& |f(x)-g(x)| \leq C_{1} \nu(d f(x))^{2}, \\
& \|d f(x)-d g(x)\| \leq C_{2} \nu(d f(x)),
\end{aligned}
$$

we get $C^{0}$-right equivalence of two $C^{1}$ mappings with locally Lipschitz differentials. But in fact the first out of the above conditions is satisfied to a very narrow class of mappings. So, without changing of the proof we can improve conditions in the above corollary to get the following

Proposition 4.2. Let $f, g:\left(\mathbb{R}^{n}, 0\right) \rightarrow\left(\mathbb{R}^{m}, 0\right)$ be $C^{1}$ mappings with locally Lipschitz differentials $d f, d g:\left(\mathbb{R}^{n}, 0\right) \rightarrow L\left(\mathbb{R}^{n}, \mathbb{R}^{m}\right)$ let $Z=\left\{x \in \mathbb{R}^{n}: \nu(d f(x))=\right.$ $0\}$, and let $0 \in Z, m \leq n$. If there exist a neigbourhood $U \in \mathbb{R}^{n}$ and constants $C>0, C_{1} \in\left(0, \frac{1}{2}\right)$ and $k>1$ such that

$$
\begin{aligned}
& \nu(d f(x)) \geq C \operatorname{dist}(x, Z)^{k-1}, \\
& |f(x)-g(x)| \leq C \nu(d f(x))^{k}, \\
& \|d f(x)-d g(x)\| \leq C_{1} \nu(d f(x))^{k-1}
\end{aligned}
$$

for $x \in U$, then $f$ and $g$ are $C^{0}$-right equivalent.

Moreover, in the proof of above theorem it was proved that the vector field $W$ is a Lipschitz mapping in $G \backslash(\mathbb{R} \times Z)$. But under the assumption of 
Proposition 4.2 we can easily prove that this vector field is also Lipschitzian in $G$. Therefore we obtain Proposition 1.4.

Analogously, we can improve [8, Theorem 2]. Namely, we have.

Proposition 4.3. Let $f, g:\left(\mathbb{R}^{n}, 0\right) \rightarrow(\mathbb{R}, 0)$ be $C^{1}$ functions such that $\nabla f, \nabla g$ are locally Lipschitz mappings. If $\nabla f(0)=0$ and there exist a neigbourhood $U$ of $0 \in \mathbb{R}^{n}$ and constants $C, C^{\prime}>0$ such that

$$
|(g-f)(x)| \leq C|\nabla f(x)|^{2}, \quad|\nabla(g-f)(x)| \leq C^{\prime}|\nabla f(x)|^{2} \quad \text { for } x \in U,
$$

then $f$ and $g$ are bi-lipschitz equivalent.

Acknowledgements. I am deeply grateful to Mihai Tibăr for his valuable comments and advices.

Open Access. This article is distributed under the terms of the Creative Commons Attribution 4.0 International License (http://creativecommons.org/licenses/ by/4.0/), which permits unrestricted use, distribution, and reproduction in any medium, provided you give appropriate credit to the original author(s) and the source, provide a link to the Creative Commons license, and indicate if changes were made.

Publisher's Note Springer Nature remains neutral with regard to jurisdictional claims in published maps and institutional affiliations.

\section{References}

[1] Bekka, K., Koike, S.: Characterisation of $V$-sufficiency and $C^{0}$-sufficiency of relative jets. https://arxiv.org/pdf/1703.07069.pdf

[2] Bochnak, J.: Relévement des jets. In: Séminaire Pierre Lelong (Analyse), (année 1970-1971). Lecture Notes in Mathematics, vol. 275, pp. 106-118. Springer, Berlin (1972)

[3] Jelonek, Z.: On the generalized critical values of a polynomial mapping. Manuscr. Math. 110, 145-157 (2003)

[4] Kuiper, N.H.: $C^{1}$-equivalence of functions near isolated critical points. In: Symposium on Infinite Dimensional Topology (Louisiana State University, Baton Bouge, 1967). Annals of Mathematics Studies, vol. 69, pp. 199-218. Princeton University Press, Princeton (1972)

[5] Kuo, T.C.: On $C^{0}$-sufficiency of jets of potential functions. Topology 8, 167-171 (1969)

[6] Kurdyka, K., Orro, P., Simon, S.: Semialgebraic Sard theorem for generalized critical values. J. Differ. Geom. 56, 67-92 (2000)

[7] Migus, P.: $C^{r}$-right equivalence of analytic functions. Demonstr. Math. 48(2), 313-321 (2015)

[8] Migus, P.: Local $C^{r}$-right equivalence of $C^{r+1}$ functions. Glasg. Math. J. 59(1), 265-272 (2017)

[9] Migus, P., Rodak, T., Spodzieja, S.: Finite determinancy of non-isolated singularities. Ann. Pol. Math. 117(3), 197-206 (2016) 
[10] Rabier, P.J.: Ehresmann fibrations and Palais-Smale conditions for morphisms of Finsler manifolds. Ann. Math. Second Ser. 146, 647-691 (1997)

[11] Rodak, T., Spodzieja, S.: Equivalence of mappings at infinity. Bull. Sci. Math. 136(6), 679-686 (2012)

[12] Takens, F.: A note on sufficiency of jets. Invent. Math. 13, 225-231 (1971)

[13] Tougeron, J.C.: Idéaux de fonctions différentiables. Ergebnisse der Mathematik und ihrer Grenzgebiete, Band 71. Springer, New York (1972)

[14] Xu, X.: $C^{0}$-sufficiency, Kuiper-Kuo and Thom conditions for non-isolated singularity. Acta Math. Sin. (Engl. Ser.) 23(7), 1251-1256 (2007)

Piotr Migus

Air Force Institute of Technology

ul. Księcia Bolesława 6

01-494 Warsaw

Poland

e-mail: migus .piotr@gmail.com

Received: 4 July 2018 